\title{
FIB/SEM Tomography of Wound Biofilm
}

Binbin Deng ${ }^{1,2}$, Kasturi Ganesh Barki ${ }^{1}$, Subhadip Ghatak ${ }^{1}$, Sashwati Roy ${ }^{1}$, David W. McComb ${ }^{2}$, Chandan K. Sen ${ }^{1}$

1. Davis Heart \& Lung Research Institute and Comprehensive Wound Center, Department of Surgery, The Ohio State University Wexner Medical Center, Columbus, OH 43210 USA

2. Center for Electron Microscopy and Analysis, Department of Materials Science and Engineering, The Ohio State University, Columbus, OH 43212 USA

Biofilm has a complex architecture and show high tolerance to host immune responses and antimicrobial agents [1]. Biofilm infection of chronic wounds often makes them very resistant to treatment, and can be a severe health problem in patients with compromised immune systems. Despite the extensive study of biofilm in the past 15 years [2], the pathogenic biofilm structures remain to be characterized. DualBeam FIB/SEM tomography is a suitable tool to investigate three-dimensional (3D) structures of biological materials at intermediate to high resolution. In order to understand the biofilm architecture in animal model and clinical chronic wound, we have studied a porcine pre-clinical wound model involving single-species and multi-species infection as well as chronic wound from a patient using FIB/SEM tomography.

Domestic Yorkshire pigs were subjected to a full-thickness burn (2"x2"). A clinically relevant mixedspecies infection was established. Patient tissue was collected from one year old chronic wound. Pig biofilm and patient wound tissue were cut into 200-500 $\mu \mathrm{m}$ thick sections. Samples were chemically fixed and en bloc stained. After dehydration and infiltration, tissue sections were embedded in durcupan resin and incubated at $60^{\circ} \mathrm{C}$ for 2 days [3]. The resin embedded tissue was trimmed by ultramicrotome and mounted on an SEM stub. FIB slice and view datasets were collected on a Helios Nanolab 600 DualBeam (FIB/SEM) (FEI, Hillsboro). Images were processed and visualized using MIPAR[4], ImageJ [5], IMOD [6], Chimera [7] and Avizo (FEI, Hillsboro) software packages.

Biofilms formed by aggregated bacteria are encapsulated in extracellular polymeric substance (EPS). Our FIB/SEM tomography results revealed structures of pig biofilm (Figure 1) and patient wound (Figure 2). 3D structures demonstrated the non-uniform distribution of biofilm. Macrophages were found engulfing bacterium. Phagocytes were found squeezing through capillary walls to reach the infection sites. These data are providing unique insights that are improving our understanding of the process of biofilm wound healing. 


\section{References:}

[1] L Hall-Stoodley et al. "Bacterial biofilms: from the Natural environment to infectious diseases", Nature Reviews Microbiology. 2004; 2(2): p95-108

[2] T Bjarnsholt "The role of bacterial biofilms in chronic infections", APMIS suppl. 2013; 121(136): p1-51

[3] AJ Bushby et al. "Imaging three-dimensional tissue architectures by focus ion beam scanning electron microscopy", Nature Protocols 2011; 6(6): p845-858

[4] JM Sosa et al. "Development and application of MIPAR ${ }^{\mathrm{TM}}$ : a novel software package for two- and three-dimensional microstructural characterization", Integrating Materials and Manufacturing Innovation 2014; 3:10

[5] CA Schneider et al. "NIH Image to ImageJ: 25 years of image analysis", Nature Methods 2012; 9(7): p671-675

[6] JR Kremer et al. "Computer visualization of three-dimensional image data using IMOD”, Journal of Structural Biology 1996; 116(1): p71-76

[7] EF Pettersen et al. "UCSF Chimera - a visualization system for exploratory research and analysis", Journal of Computational Chemistry 2004; 25(13): p1605-1612

[8] The authors acknowledge funding from Ohio Third Frontier Program, The Institute of Materials Research and The Ohio State University"

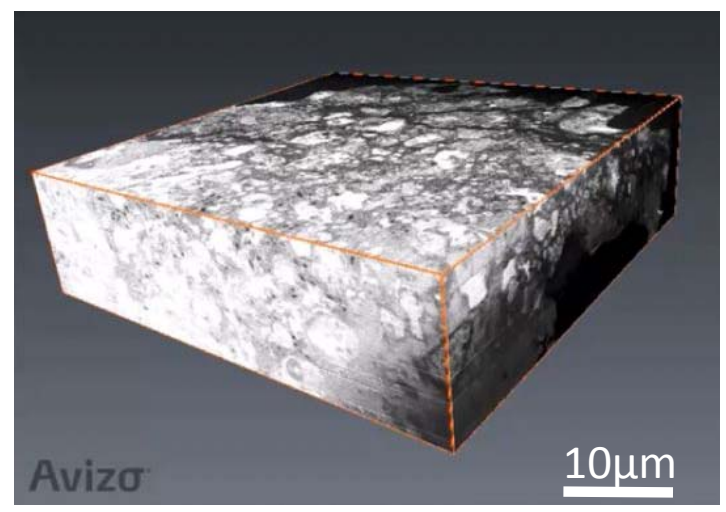

Figure 1. 3D volume views of pig biofilm obtained using FIB/SEM tomography

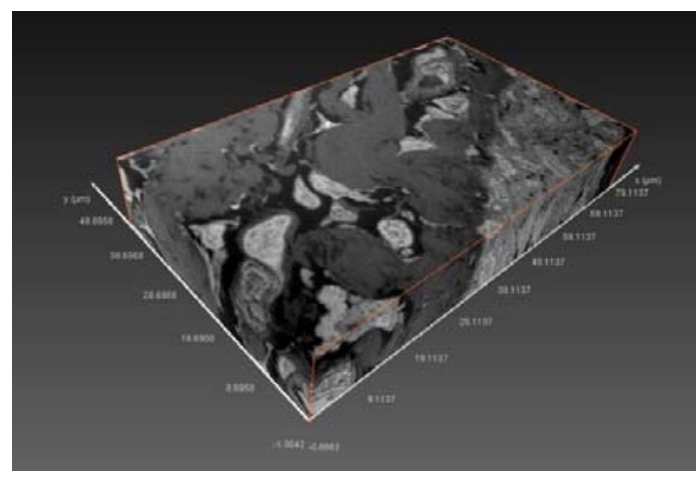

Figure 2. 3D volume views patient wound obtained using FIB/SEM tomography 